\title{
A New Way to Use Wikipedia in Education: A Pilot Study of Map-like Wikipedia Visualization on iPad
}

\author{
Cheong-Iao Pang, Sek-Si Si, and Sio-Kuan Chio
}

\begin{abstract}
Pad has started to appear in classrooms recently. Studies show that the tablet computer engages students in learning and improves student performance. On the other hand, Wikipedia attracts many attentions in education field as well despite of worries about credibility and reliability. We have developed a prototype of interactive Map-like Wikipedia Visualization in an earlier research project. In order to test the feasibility of such a new technology in classrooms, we conducted an explorative study which combines the usage of iPad, Wikipedia and the visualization in a high school in Macau S.A.R. This paper presents the design of this study and the result we observed.
\end{abstract}

Index Terms-iPad, tablet computer, high school education, Wikipedia, map-like visualization, information visualization

\section{INTRODUCTION}

In recent years Apple's iPad not only obtains a great success in the consumer electronic market, but also gains the attentions from schools and education providers in Macau S.A.R. The local government provides funding and support to high schools and encourages them to purchase iPad. As iPad starts to appear in classrooms, teachers need to re-design their classes and make use of iPad.

On the other hand, Wikipedia, with content contributed by volunteers worldwide, has become the most well-known free online encyclopedia [1]. Although some people worry about the quality of its articles and the neutrality of certain topics, some people start to use it in lectures as well as encourage students to interact with it.

In many circumstances we face in education, we instruct students to read about articles of a particular domain. For instance, students are required to read through facts and figures about Asian countries on Wikipedia before writing essays assigned by a Geography teacher. The good news is Wikipedia has its own category system to group similar articles together in one or more categories. However, the native Wikipedia web interface may not be friendly enough to optimize users' access to these categories. Users need to "guess" in which category an article may fall, scroll down to the bottom of the page, and then click on links to see what

Manuscript received March 5, 2013; revised May 3, 2013. This work was supported by the Macau S.A.R. - Science and Technology Development Fund under Grant 021/2011/A.

Cheong-Iao Pang is with the University of Macau, Macau S.A.R. and is currently a PhD student of The University of Melbourne, Australia (e-mail: inbox@patrickpang.net).

Sek-Si Si and Sio-Kuan Chio are with The Workers' Children High School, Macau S.A.R (e-mail: dickson@seesix.com, kuansio@yahoo.com.hk) other contents are related. Alternatively, they can select a top-level category (e.g. Science, Arts, Geography, etc.) in the main page, repeatedly navigate down to sub-categories and hopefully reach the correct place at one point.

Neither of the abovementioned use-cases is satisfactory. Therefore, Map-like Wikipedia Visualization was introduced to display the category system in a 2D map metaphor. The visualization presents categories and related sub-categories in a way that is similar to a geographical map, which shows countries, states, and counties in different colors, fonts and styles. In this way, it will be easier to discover the concerned category and the related content in one view.

Combining the usage of iPad, Wikipedia and Map-like Visualization in classrooms is a new idea. We attempted to integrate these as an activity to chemistry classes on the Form 3 students in one high school in Macau S.A.R. A preliminary study was conducted to investigate the student performance and perception of using these new technologies in the class.

The remainder of this paper presents the related work, the design of the class activity, the result of the study and discussions on the observations.

\section{RELATED WORK}

\section{A. Using Tablet Computers in Classrooms}

Since the introduction of tablet computers, research was carried out to study the possibility of using them in the education domain. Enriquez discovered that students showed overwhelmingly positive attitudes on their learning experience while using tablet computers in the classroom environment. The interactive learning environment was also found to improve student performance compared with the traditional instructor-centered learning environment [2].

Previous studies searched for ways to use iPad in lectures. Research showed case studies of using iPad in various courses such as Music, English and etc. A survey was conducted to reveal students' perceptions on iPad. The result showed that iPad helped them learn the course content and apply the course content to solve real world problems. Students showed higher engagement in courses taught with iPad. The majority of students presented higher motivation and participation in classes taught with iPad, and agreed tablets were more convenient than desktop and laptop computers [3]. Another study reflected that students identified unique visual and aural learning opportunities offered by activities using mobile tablet. They engaged in personal and immediate feedback from the tablet, from which a better learning environment was created [4]. 


\section{B. Using Wikipedia in Education}

Many concerns about Wikipedia are raised, for instance, its quality issues when people suggest using it in lectures. Actually, the quality of Wikipedia was compared and found close to that of Encyclopedia Britannica [5]. However, another study showed that Wikipedia was not a totally reliable resource [6]. Nevertheless, even Wikipedia itself claimed that "Wikipedia may not be ideal as a source for all academic uses, and (as with other sources) suggests that at the least, one strength of Wikipedia is that it provides a good starting point for current information on a very wide range of topics." [7]

Research has studied different method to utilize Wikipedia in teaching. Moy et al. conducted a class project that allowed students to learn and explore chemistry in Wikipedia. They observed students were more critically comparing materials to those in normal class, which helped students to develop a higher level of explanatory ability and knowledge [8]. Konieczny suggested instead of banning Wikipedia in class, students should be taught to use it with caution and properness. Eventually students will benefit from the tool whereas it also enhances teachers' portfolio of their teaching strategy [9].

Wikipedia brings benefits to students according to previous studies. Patten and Keane carried out integration of Wikipedia projects in two courses. Students reflected generally positive feedback, for example, Wikipedia was informative, helped them to learn a lot and improved their writing and research skills [10]. After five years of the publishing of Konieczny's work [9], the author summarized the teaching experience with Wikipedia, and discussed ways to incorporate Wikipedia into the curriculum as well as common problems faced in daily teaching [11]. This demonstrated the feasibility of applying Wikipedia in general classrooms in this digital era.

\section{Map-like Wikipedia Visualization}

An advantage of geographic maps is that they can be easily comprehended. Elements such as mountains, valleys, land, rivers, sea, etc. are readily recognized by people even without special training. Representing complex data in the form of a map enables people to relate to such representations more easily at the first glance on the visualization. Skupin presented a method that produces a map-like visualization for a document corpus [12]. Similarly, Pang and Biuk-Aghai used the same concept to produce a map-like visualization for Wikipedia [13].

It is relatively inconvenient to look up related information in a particular domain in Wikipedia even though there is a category system which groups similar pages together. Users have two ways to access the category system. They can make a "guess" and enter a page that falls in that category, and scroll down to the bottom to see the category list. Alternatively, they need to click on a top-level category (e.g. Science, Arts, Geography, etc.) then try to locate targeted sub-categories down level by level. Wikipedia is indeed designed for accessing one page quickly by a keyword search and then travel to other pages with the inter-links embedded in the content, and perhaps this makes categorization support less important and gain less attention.

Map-like Wikipedia Visualization aims to address the difficulty in category navigation. It analyzes the category system in Wikipedia and presents the categories in a $2 \mathrm{D}$ image using a map metaphor. A map metaphor means using lines, colors, font sizes and the way of showing hierarchy that are similar to the ones we see in a geographical map. Fig. 1 shows a part of the entire visualization.

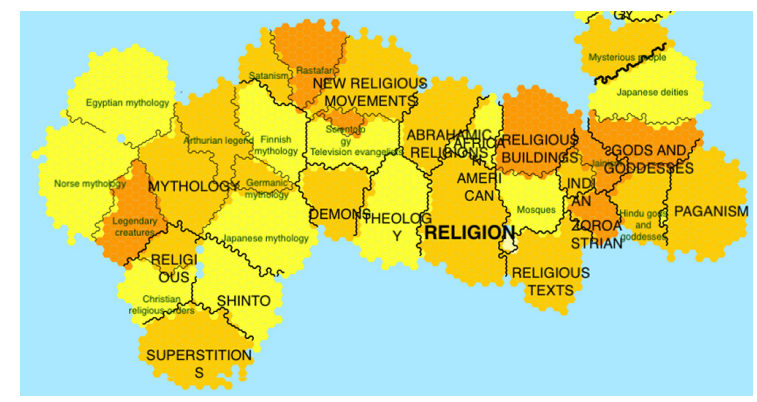

Fig. 1. Map-like wikipedia visualization

As shown in Fig. 1, top-level categories (i.e. Religion) is shown in largest font size and in bold, whereas lower sub-categories are placed around and displayed using a smaller font size. The amount of content inside a category is represented by different colors. The darker the color, the more articles lie under that category, and vice versa.

The positioning of each item is determined by the similarity (i.e. relationship) to other items in the visualization. The value of similarity is computed with how much content is shared between two categories at the same time [14]. The more shared content means they are more related to each other and the algorithm tries to place them nearer. In this way, related categories are positioned closely and users can better spot relevant information easier.

\section{DESIGN OF THE STUDY}

We conducted a pilot study to observe the usage of iPad and Wikipedia in high school education. The study was supported by a high school in Macau S.A.R. Apple iPad has been being used for a period time in this school so teachers, students and supporting staffs are familiar with the operation of iPad. We selected to implement the study in the chemistry classes of Form 3 (the third year of Macau high school; aged 15 in average) students. Totally seven classes (240 students) were involved in this activity.

iPad tablets are often used in the class for reference purpose in this school. Students are required to look for information about a subject, for instance, chemistry, according to teacher's instruction. They are recommended to read articles in the Chinese Wikipedia (http://zh.wikipedia.org/) and report their findings. The information in Wikipedia is regarded as external reading to students. Teachers will discuss and explain the topic with students in later classes.

This study aimed to compare the student performance and perception between the original Wikipedia web interface and the new map-like visualization for Wikipedia. Students were given a question sheet with three questions which cover knowledge neither taught before nor included in the textbook. 
Students needed to find answers from the Wikipedia and were not allowed to use other materials and websites. iPad devices were connected to a restricted wireless network to limit access to other websites. The three questions are listed as follows (translated from Chinese):

- List all the awards of Nobel Prizes.

- Name two halogen elements and find out what naming principle are used when choosing their Chinese names.

- List 10 Chinese chemistry scientists.

Question 1 and 3 are more specific questions while question 2 is a relatively open-end question. We expect the new visualization is good at open-end questions as it allows students to see and explore more information when they navigate. This design tries to verify this assumption.

Students were divided into two groups and every student was given an iPad. One group used the original Wikipedia website in a modified browser app, which recorded down the number of pages read and the navigation history. The other group used the map-like visualization app to locate the answer. Both apps automatically tracked down users' operations and recorded the browsing histories and statistics. These statistics include numbers of moves, zoom-in/out, sub-categories visited and pages opened. Similar metrics are suggested in [15] to evaluate an interactive visualization. We also recorded the time needed for completing the question sheet in both groups.

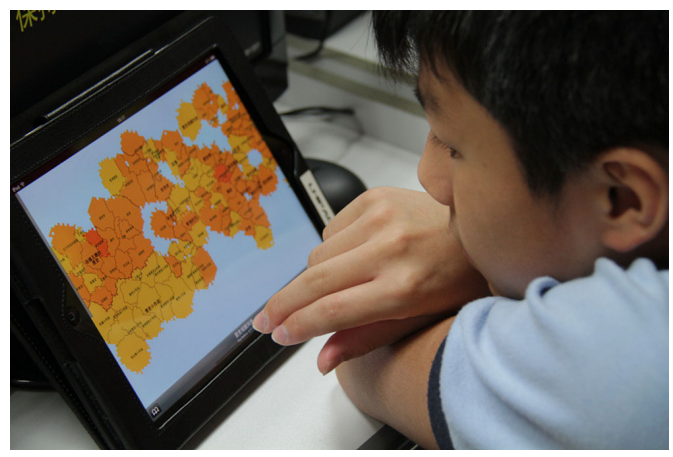

Fig. 2. A student exploring map-like wikipedia visualization

Before the start of the study, a 10-minute introductory video was played to all students. This video clip demonstrated how to operate the Wikipedia website as well as the map-like visualization. Students were then given about 40 minutes to work on the question sheets. Figure 2 shows a student using touch gestures to explore the map-like visualization. The entire activity lasted for 50 to 60 minutes.

Completed question sheets were assessed by teachers. A score ranging from 0 to 10 was given for each answer sheet to represent the accuracy of the answers. Furthermore, students were randomly selected and interviewed. They reported their experience on the application they used. Finally, the teachers also reported their observations on students, and expressed their comments on both the visualization and the activity.

\section{RESULTS}

This section briefly describes the quantitative results obtained from the answers of students and the statistics recorded, which consists of the accuracy, the interaction in both apps and the elapsed time.

\section{A. Accuracy}

As Table I shows, the students using the native Wikipedia website have a slightly higher average score, i.e. better accuracy, than those using map-like visualization. In terms of the lowest score and the standard deviation of scores, the students using the website seem to perform more steadily in this study. However, in both groups there are students getting the highest mark. It is also notable that there are slightly more students of good performance (scored greater or equal 9.0 points) from the map-like visualization group. Before the experiment, it was suspected that negative impact would result in student performance for those who use the map-like visualization, but the result shows the impact is not obvious

TABLE I: ACCURACY (SCORE) OF STUDENT ANSWERS

\begin{tabular}{lll}
\hline \hline & $\begin{array}{l}\text { Map-like } \\
\text { Visualization }\end{array}$ & $\begin{array}{l}\text { Wikipedia Web } \\
\text { Interface }\end{array}$ \\
\hline Average & 6.22 & 6.93 \\
Minimum & 0.0 & 1.3 \\
Maximum & 10.0 & 10.0 \\
Standard Deviation & 2.13 & 1.41 \\
Number of Students & 4 & 3 \\
Scored $>=9.0$ & & \\
\hline \hline
\end{tabular}

\section{B. User Interactions}

TABLE II: USER INTERACTION STATISTICS (IN AVERAGE)

\begin{tabular}{lll}
\hline \hline & $\begin{array}{l}\text { Map-like } \\
\text { Visualization }\end{array}$ & $\begin{array}{l}\text { Wikipedia Web } \\
\text { Interface }\end{array}$ \\
\hline Moves & 176.83 & --- \\
Zoom-in & 13.94 & --- \\
Zoom-out & 11.04 & --- \\
Sub-categories Opened & 6.67 & --- \\
Number of Queries & --- & 22.29 \\
Number of Link Follows & --- & 18.19 \\
Pages Read & 45.73 & 46.96 \\
\hline \hline
\end{tabular}

Table II presents the average interaction statistics recorded from the iPad app. The first four metrics are available for the map-like visualization only. Moves indicates the number of movements triggered by finger touches for viewing different part of the image. Zoom-in/out means the number of attempts to enlarge or shrink the visualization to show/hide details. The number of sub-categories opened equals the number of sub-category clicked to show content inside. The data of number of queries and link follows are recorded from the web interface. Number of queries is the number of searches performed by a student, whereas number of link follows is the number of times that a student clicks on one hyperlink and navigates to another Wikipedia article. Finally, the figure of pages read represents how many articles are opened and read by a student.

From the figure in Table II, we conclude that the numbers of pages read are similar in both groups of students. For the students using the map-like visualization, the times of zoom-in and zoom-out are close, which reflects students' action of zooming-in to explore detail information and zooming-out to higher abstract level to continue searching, 
rather than staying at the same level. On the other hand, the figures show that students using webpage interface make use of both the search engine and inter-page links to navigate within Wikipedia.

\section{Time Elapsed}

The average completion time for the students using visualization is 23.73 minutes while 23.44 minutes for the other group, which shows minimal difference only. If students are classified into 10-minute time-slot with their completion time, we observe that more students $(27 \%)$ from the group of native Wikipedia finish faster (within 20 minutes), in contrast to only $19 \%$ in another group. Nonetheless, $3 \%$ web interface users complete very slow while no one using the map-like visualization needs over 40 minutes to finish.

TABLE III: COMPLETION TIME OF BOTH GROUPS

\begin{tabular}{lll}
\hline \hline & $\begin{array}{l}\text { Map-like } \\
\text { Visualization }\end{array}$ & $\begin{array}{l}\text { Wikipedia Web } \\
\text { Interface }\end{array}$ \\
\hline 0-10 Minutes & $3 \%$ & $5 \%$ \\
10-20 Minutes & $16 \%$ & $22 \%$ \\
20-30 Minutes & $71 \%$ & $61 \%$ \\
30-40 Minutes & $9 \%$ & $9 \%$ \\
Over 40 Minutes & $0 \%$ & $3 \%$ \\
\hline \hline
\end{tabular}

\section{DisCUSSIONS}

\section{A. Towards Interactive and Mobile Education}

Students showed high engagement and interest in iPad during this study. In the discussion with students, many of them agreed applying iPad in class was an advance in daily teaching. The knowledge became more solid and impressive. Besides, the mobility of tablet computers also brings convenience to students as they needed to access computer rooms to have interactive course material in the past. We believe high school students accept having new electronic devices such as iPad to be used in their school lives, however, the key is on the apps. Without appropriate apps as teaching materials, iPad or other tablet computers are nothing more than gaming and Internet devices. From this point of view, studies and experiments on interactive visualization and tools, for example the one we are doing, not only contributes to the computer science discipline but also gives new directions to educators.

\section{B. Enabling Exploratory Search}

As reported in the last section, the students in the map-like visualization group used moving, pinching and spreading gestures to explore the categories. In the process, they repeatedly analyzed the names of items, determined the relevance to the questions, read for more details in deeper levels, and then return back to higher levels to continue searching. In fact, the seeking behaviors of the students fall in a type of exploratory search.

Exploratory search is an information seeking process which emphasizes learning and investigating activities. It involves cognitive processing and interpretation on the result in multiple iterations. The seeker needs to spend time to read and make sense of encountered information. New knowledge is developed and acquired during the process [16]. When people face unfamiliar topics, they generally perform exploratory searches. During the process, it is likely that the problem will become better understood [17]. In fact, the student learning process is comparable to this paradigm: students know little knowledge before taking classes, and we expect they can have a better understanding on the knowledge after class.

With the elements of learning and investigating, tools supporting exploratory search, such as our visualization software, are beneficial to students. It broadens students' eyesight by exposing more information at one time, along with encouraging critical thinking and improving analytic skills during the knowledge acquisition process. One teacher commented that answers showed more variety for the open-end question (Q2) from the group using the map-like visualization. He added that students stopped searching after the Wikipedia search engine returned a seemingly accurate result, whereas the visualization created more chances for students to look deeper.

"In the progress of finding information, I need to think about different aspects of the target. Finally I realize that I learn much more afterwards." (Student 1)

"I can read more other information of one target [in the map-like visualization]." (Student 2)

According to feedback from student 1 and 2, even they did not know the terminology of exploratory search, but they discovered similar facts and advantages.

"This level-by-level searching is more suitable for higher grade students or adults as they know clearer about categories." (Student 9)

We agree the view of student 9. Since exploratory search requires cognitive and analytical abilities, younger students may not be capable to handle. Moreover, analysis and critical thinking need common sense and domain knowledge to support. We expect senior students, because of possessing deeper knowledge and clearer visions, will perform better in this kind of exploratory search activities.

\section{Supplement to Traditional Searching}

"It [map-like visualization] is slower than the old approach." (Student 5)

"If I have time, I will use this [map-like visualization]. Otherwise I will go to Google and make a search directly." (Student 8)

Many students reported longer time is needed to use the map-like visualization. The figure of Table III in Section IV also demonstrates the similar phenomenon. In many cases, students preferred to use an online search engine because the search results show up in just a few seconds and are more specific. Small amount of students also commented search engines are familiar to them but the visualization is not. They needed to spend time to get used to the map-like software which caused slower performances. 
Educators have a different point of view though. Participated teachers suggested that this kind of software is another direction to break the "monopoly" of people's searching habits. We now depend on what online search engine provides to us in the result, which appearance might be manipulative out of commercial or political concerns, and most of time the result is tightly bounded by the search terms. It goes against the idea of encouraging students to think out of the box. In contrast, during the use of this explorative visualization, students have more chances to see relevant information thus build up a mindset of taking comprehensive understanding to real-world problems. This is important for the current students to survive in the rapidly changing world. Nevertheless, long-existed search engines are still having their values in both our daily lives and education. New technologies, such as the exploratory search systems, can be seen as a supplement to the traditional web searching.

\section{Technical Issues}

We faced some technical issues in the pilot study. Firstly, transporting $40 \mathrm{iPad}$ across classrooms is an issue. Considering each iPad weighing 650 grams, 40 iPad tablets then weigh 26 kilograms in total. A special trolley with a cabinet was purchased to store iPad tablets. The trolley also features recharge cables for charging the batteries. Installing applications and configuring wireless network on these iPad are tedious tasks which are time consuming and require need considerable manpower. At the time of the study, technicians need to repeat these tasks manually on every machine. In the future, we hope that the tablet vendors will support broadcasting these settings in a batch.

\section{CONCLUSION}

This paper shows an application of Map-like Wikipedia Visualization and Wikipedia in a high school. Feedback from both students and teachers are positive and encouraging. Students generally engage in learning with the visualization on iPad. The study also suggests the existence of exploratory search in the seeking behavior of students and the support of exploratory search with the interactive map-like visualization. In general, we believe the integration of our tools in classes will help train students on their analytical and critical thinking.

This study is limited to students in the same year and one subject only. There may be other findings when applying the visualization to other students. In the future, our research will focus on improving the visualization and trying to conduct more studies on other students.

\section{ACKNOWLEDGMENT}

We gratefully acknowledge the support for this research from the Macau Special Administrative Region - Science and Technology Development Fund under grant 021/2011/A. We also appreciate the principal Mr. Jiezhao Zheng, the teachers (especially Ms. Pui-Ieng Tai and Mr. Pou-Weng Long) and technicians of The Workers' Children High School, Macau S.A.R, for providing assistance and support on this project.

\section{REFERENCES}

[1] D. O'Leary, "From each according to his knowledge," Computer, vol. 41, no. 2, pp. 34-41, Feb. 2008

[2] A. G. Enriquez, "Enhancing student performance using tablet computers," College Teaching, vol. 58, pp. 77-84, Aug. 2010.

[3] W. Miller, "iTeaching and learning," Library Technology Reports, vol. 48, no. 8, pp. 54-59, Nov. 2012.

[4] J. P. Rossing et al., "iLearning: The future of higher education? Student perceptions on learning with mobile tablets," Journal of the Scholarship of Teaching and Learning, vol. 12, no. 2, pp. 1-26, 2012.

[5] J. Giles, "Internet encyclopaedias go head to head," Nature, vol. 438, pp. 900-901, Dec. 2005.

[6] Thomas Chesney, "An empirical examination of Wikipedia's credibility," First Monday, vol. 11, no. 11, Nov. 2006.

[7] Wikipedia. Reliabily of Wikipedia. [Online] Available: http://en.wikipedia.org/wiki/

[8] C. L. Moy, "Improving science education and understanding through editing Wikipedia," Journal of Chemical Education, vol. 87, no. 11, pp. 1159-1162, Aug. 2010.

[9] P. Konieczny, "Wikis and Wikipedia as a teaching tool," International Journal of Instructional Technology \& Distance Learning, vol. 4, no. 1, 2007.

[10] K. Patten and L. Keane. (2012). Integrating Wikipedia projects into IT courses: does Wikipedia improve learning outcomes. [Online] Available: http://aisel.aisnet.org/amcis2012/proceedings/EndUserIS/28

[11] P. Konieczny, "Wikis and Wikipedia as a teaching tool: five years later," First Monday, vol. 17, no. 9, Sept. 2012.

[12] A. Skupin, "The world of geography: visualizing a knowledge domain with cartographic means," in Proc. of the National Academy of Sciences, vol. 101, pp. 5274-5278, Apr. 2004.

[13] C. I. Pang and R. P. B. Aghai, "Wikipedia world map: method and application of map-like wiki visualization," in Proc. of the 7th International Symposium on Wikis and Open Collaboration, Oct. 2011.

[14] C. I. Pang and R. P. B. Aghai, "A method for category similarity calculation in wikis," in Proc. of the 6th International Symposium on Wikis and Open Collaboration, Jul. 2010.

[15] J. Scholtz, "Beyond usability: evaluation aspects of visual analytic environments," in Proc. of IEEE Symposium on Visual Analytics Science and Technology, 2006.

[16] G. Marchionini, "Exploratory search: from finding to understanding," Communications of the ACM, vol. 49, no. 4, Apr. 2006.

[17] R. W. White and R. A. Roth, "Exploratory search: beyond the query-response paradigm," Synthesis Lectures on Information Concepts, Retrieval, and Services, pp. 1-98, 2009.

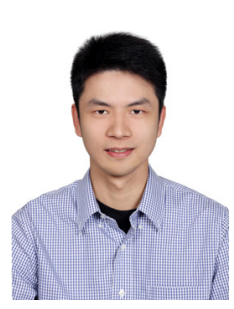

Cheong-Iao Pang received his MSc degree in Software Engineering from the University of Macau, Macau S.A.R. in 2011. He is currently pursuing a $\mathrm{PhD}$ degree in the Department of Computing and Information Systems at the University of Melbourne, Australia. His research interests include information seeking behavior, health information seeking, information visualization and Wikipedia related applications.

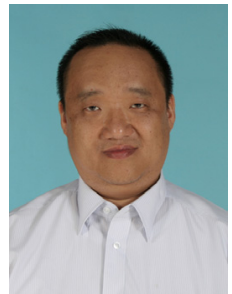

Sek-Si Si received his bachelor degree from South China Normal University, China. He is the Director of Center for Multimedia-assisted Teaching in The Workers' Children High School, Macau S.A.R. since 1998. He obtained 22 awards in the Award Scheme on Instructional Design since 1999, which is organized by Education and Youth Affairs Bureau (DSEJ). He also presented two papers in educational symposiums co-organized by the University of Macau and Macau

S.A.R. Government

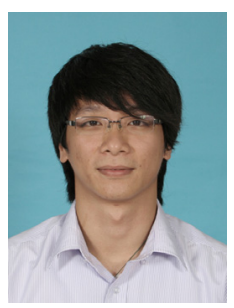

Sio-Kuan Chio received his BSc degree from the University of Macau, Macau S.A.R in 2008. He is currently a laboratory technician for multimedia teaching in The Workers' Children High School, Macau S.A.R. He is pursuing an MSc degree in the University of Macau at the moment. 